\title{
MUNICIPALIZAÇÃO DA VIGILÂNCIA EPIDEMIOLÓGICA: UMA PROPOSTA EM CONSTRUÇÃO
}

Denise Schout*

RESUMO:: Neste artigo serão apresentados aspectos do processo de municipalização das atividades de vigilância epidemiológica no Estado de São Paulo, os entraves que o Sistema de Vigilância Epidemiológica têm na sua estrutura que dificultam a efetiva inclusão destas atividades pelos Municipios e quais as perspectivas existentes para sua superação.

A análise $e$ as propostas para o enfrentamento das dificuldades existentes na municipalização tem o intuito de contribuir para o desenvolvimento deste processo na direção da incorporação da epidemiologia nas práticas de saúde e resultam da experiência da autora em algumas das instâncias do Sistema, como médica sanitarista atuando na coordenação de serviços de vigilância epidemiológica.

\section{INTRODUÇÃO:}

A reestruturação da Secretaria de Estado da Saúde em 1985/86 e a implantação do Sistema Unificado e Decentralizado de Saúde (SUDS), com a estadualização e municipalização das ações de saúde durante os anos de 1987/88, em todos os Municipios do Estado de São Paulo, com exceção da Capital,

"Médica Sanitarista. OMBUDSMAN - editora de qualidade da Superintendência de controle de Endemias - SUCEN 
determinaram mudanças no papel das instituições envolvidas neste processo, sem que houvesse amadurecimento adequado destas para a incorporação de novas atribuições, de forma que não implicasse em descompasso nas atividades e programas $(1)$.

A legislação na área da Saúde, concretizada na Constituição de 1988 e através da promulgação da Lei orgânica de saúde (lei no. 8080/90), representou um grande avanço e traduziu os anseios e a discussão acumulada na área de Saúde Coletiva a respeito da estrutura de saúde existente em nosso meio(2). A criação do Sistema Único de Saúde (S.U.S.) reforçou uma organização dos serviços de saúde com direção única em cada esfera do governo e estruturado em nível municipal com regionalização e hierarquização de ações (3).

A municipalização era, e ainda é, uma proposta que traz os avanços necessários na estrutura de prestação de serviços de saúde, para melhor dar conta do quadro nosológico observado em nosso meio.

Em maio de 1987, são assinados os primeiros convênios SUDS entre o Ministério da Saúde. Previdência e Assistência Social, Educação e 11 governos estaduais, dentre os quais o Estado de São Paulo(4). A implantação e o desenvolvimento do SUDS, gerenciado pelo Secretário de Saúde do periodo (1987/1990), esteve orientado por interesses político-partidários, o que se refletiu dentro da estrutura da Secretaria em vários aspectos ${ }^{1}$, dos quais destaca-se:

1. A indicação para as diretorias dos Escritórios Regionais de Saúde (ERSAs), criados no final da gestão anterior, de elementos pertencentes às forças politicas que compunham o governo, que na maioria das vezes não tinham formação técnica especifica para exercerem este cargo. 
2. A extinção da carreira de médico sanitarista (1987), com a indicação das chefias das unidades de saúde por critério predominantemente político e não mais por escolha pública e de acordo com a carreira anteriormente existente.

Isto refletiu-se no processo de municipalização, onde a discussão técnica, a formação de recursos humanos nos Municipios e a incorporação das atividades, foi dominada pelo jogo político-partidário e não pelo planejamento das ações baseado no diagnóstico epidemiológico dos Municípios $(2,5)$.

A Vigilância Epidemiológica também sofreu modificações neste periodo, com a criação do Centro de Vigilância Epidemiológica em dezembro de 1985. Este órgão que passou a coordenar o sistema de Vigilância Epidemiológica, foi pressionado neste processo a descentralizar suas atividades e adaptar o sistema à nova realidade.

\section{O SISTEMA DE VIGLÂNCIA EPIDEMIOLÓGICA}

O sistema de Vigilância Epidemiológica foi criado, em nível nacional, pela Lei $n^{\circ} 6.259$ e pelo Decreto $n^{\circ} 78.231$, que o regulamentou, definindo as diretrizes e o leque de doenças objeto de notificação compulsória abarcados pelo sistema(6).

No Estado de São Paulo, foi criado em 1978 o Sistema Estadual, coordenado naquele momento pelo Centro de Informações de Saúde (CIS) o qual produziu o primeiro Manual de Vigilância Epidemiológica - Normas e Instruções. Neste manual registra-se a seguinte conceituação: 
"Entende-se por Vigilância Epidemiológica o alerta permanente e responsável em relação à ocorrência e distribuição das doenças e dos fatores ou condições que propiciem aumento do risco de transmissão ou da gravidade das doenças.

Deve-se também tornar bem clara a distinção entre a notificação, com caráter estatístico, dos dados de morbidade, que freqüentemente se torna rotineira sem conduzir à ação, e a Vigilância Epidemiológica, que representa a prática da Epidemiologia em Saúde Pública, com o objetivo de ampliar de modo oportuno e efetivo o controle das doenças.

A Vigilância Epidemiológica é portanto um pré-requisito para os programas de prevenção e controle e compreende todas as atividades necessárias para a aquisição dos conhecimentos que devem fundamentar tais programas.

(...) A Vigilância Epidemiológica é pois um sub-sistema de informaçãodecisão-controle de doenças especificas, que fornecem recomendações, avalia as medidas de controle e serve de base para o planejamento;" (6)

O sistema de Vigilância Epidemiológica então implantado estava centrado nas unidades de saúde que tinham a ação executiva; isto é, sua atribuição era realizar a notificação dos casos de Doenças de Notificação Compulsória (DNC) atendidos na unidade e nos serviços existentes na sua área de jurisdição, a investigação epidemiológica dos casos suspeitos e a execução das ações de controle. Previa-se também a análise da situação epidemiológica na sua área. $O$ nivel regional respondia pelo fluxo de informações das unidades de sua área de abrangência, consolidação e análise de dados, capacitação de recursos humanos e deveria garantir os recursos materiais necessários à execução das ações 
desenvolvidas pelo nível local. Ao nivel central competia a supervisão das atividades previstas para os diversos níveis, capacitação de recursos humanos, consolidação e análise de dados, a normatização das atividades para as doenças sob vigilância e a definição de estratégias de intervenção para o controle de doenças no âmbito de abrangência do Estado(7).

Com a criação do Centro de Vigilância Epidemiológica (C.V.E.) em 1985 (8), o Sistema toma um impulso importante na direção do aprimoramento científico das normas de Vigilância e da utilização da análise epidemiológica como base para o desenvolvimento dos programas de controles existentes. O órgão é subordinado diretamente ao Secretário, possui, além da Diretoria e Assistências, uma Central de Vigilância Epidemiológica funcionando em regime de plantão de 24 horas e 27 grupos de Vigilância (Divisões), sendo 10 com atuação no nível central e 17 responsáveis pelas ações de Vigilância nos Departamentos regionais.

As divisões centrais foram organizadas por grupos de doenças segundo a forma de transmissão (transmissão por vetores, respiratória e hídrica): uma equipe responsável pelo controle e coordenação das imunizações; um grupo para desenvolvimento de métodos e pesquisas em epidemiologia e 5 responsáveis, cujas atividades estavam voltadas para coordenar o trabalho de vigilância junto às coordenadorias (CSC, CAH, CSM, CSTE, CPMS) ${ }^{* *}$

O C.V.E. previu os 17 cargos em nível regional, os quais foram preenchidos por indicação da direção do órgão, com o aval dos diretores dos

\footnotetext{
* CSC - Coordenadoria de Saúde da Comunidade CAH - Coordenadoria de Assistência Hospitalar CSM - Coordenadoria de Saúde Mental 
Departamentos de Saúde e baseado na formação técnica específica destes elementos.

O CVE passa a coordenar o sistema e, num primeiro momento, até 1988, não é o responsável pela informação (consolidação de $S V E_{3}$, fichas, etc.)(7). Acreditava-se naquela fase inicial ser possivel o desvinculamento entre o sistema de informação e a análise epidemiológica propriamente dita. Era operacionalmente mais fácil manter-se o fluxo de informações para o C.I.S e através de um bom entrosamento, receber as informações que as equipes haviam definido como essenciais para a análise e acompanhamento epidemiológico. Considerava-se ainda, fundamental para a construção do órgão no perfil almejado, a capacitação dos elementos das equipes centrais, tornando-os especialistas nas respectivas áreas de atuação e referência para os niveis regionais, o que exigia investimento, disponibilidade de tempo dos técnicos e priorização das atividades de análise epidemiológica. A meta era elaborar relatórios sobre o comportamento das doenças visando aprimorar as estratégias de controle.

Rapidamente constatou-se que o entrosamento entre os órgãos envolvidos não era fácil, o que obrigou as Divisões do C.V.E. a trabalharem a maior parte do tempo com consolidados de SVE3 - relatórios de suspeitos, sem informações mais detalhadas. O descompasso entre a qualidade da informação e a necessidade de aprimoramento e aprofundamento das análises acabou gerando grande empenho na busca ativa de dados diretamente pelas equipes em outras fontes de informação, como o Núcleo de Epidemiologia do Hospital Emílio Ribas, a Central Médica e os responsáveis de Vigilância regionais.

Conseguiu-se o fortalecimento do órgão dentro da Secretaria, garantindo avanços significativos na qualidade das investigações realizadas, 
decorrentes do grande investimento em capacitação de recursos humanos neste período. Além disso, os "responsáveis de Vigilância Epidemiológica", como foram denominados, propiciavam e facilitavam a interlocução entre o nivel central e os diretores regionais, permitindo priorização das atividades de vigilância no nível local. A sensação vivida pelo sistema foi de aumento da agilidade das ações, efetiva troca de informações com retorno dos dados ao nivel local e valorização dos dados coletados na elaboração de estratégias de intervenção.

Com a reestruturação da Secretaria e a criação dos ERSAs não foram previstos cargos na nova estrutura para Vigilância Epidemiológica. E como o número de ERSAs ${ }^{(63)}$ era muito maior que o número de departamentos regionais (17), os antigos "responsáveis", foram gradualmente absorvidos e os novos passaram a ser indicados pelos diretores dos Escritórios Regionais. O C.V.E. também se reorganiza gradativamente, criando novas divisões. Em lugar dos antigos responsáveis pelas Coordenadorias, surgem a Divisão de Infecção Hospitalar, a Divisão de Crônicas, a Divisão responsável pelas Doenças Sexualmente Transmissiveis, AIDS e Hanseniase, a Divisão de Tuberculose e a Divisão do Meio Ambiente e Saúde do Trabalhador.

Considerando que os ERSAs nascem na mudança de administração da Secretaria, com maior influência político-partidária na designação dos administradores regionais, a área de vigilância sofre também neste processo, perdendo parcela de poder e de interferência na priorização das atividades dos programas de controle de doenças de notificação compulsória.

É neste contexto que a municipalização apresenta-se para o sistema de Vigilância Epidemiológica, exigindo do CVE redimensionamento diante da nova situação. 


\section{MUNICIPALIZAÇÃO DA VIGILÂNCIA EPIDEMIOLÓGICA}

A proposta de municipalização e sua concretização ocorram num curto espaço de tempo, o que imprimiu inúmeros problemas ao processo.

Todo o esforço empreendido pelo CVE na formação e capacitação de recursos humanos no nivel regional (DRSs) havia que ser refeito para as equipes de ERSA e para as equipes municipais, recém empossadas da responsabilidade pela vigilância em seu Municipio.

Os Municipios, por sua vez, tinham a responsabilidade pela assistência médica curativa e pelo atendimento de urgência e atuavam essencialmente nestas atividades

A incorporação dos programas de prevenção e controle nas unidades municipais, as atividades de Vigilância Epidemiológica - ressaltadas as ações de investigação epidemiológica, imunização e intervenção no meio - impuseram aos Municipios uma dinâmica não conhecida anteriormente e para a qual não haviam recursos humanos preparados e nem materiais previstos.

Esta situação foi enfrentada de forma heterogênea no Estado, isto é, onde existiam diretores e equipes regionais capazes e direções municipais competentes a incorporação processou-se gradualmente, com capacitação de recursos humanos, garantindo uma acomodação, com reflexos positivos na assistência prestada pelos serviços municipais e na situação epidemiológica do Município. Porém, isto não aconteceu na maioria dos Municípios e, na verdade, não foi reflexo de uma política especifica, mas fruto da casualidade. 
A maioria dos Municípios não incorporou na sua rede os programas de prevenção e controle e restringiu as atividades de Vigilância Epidemiológica ao fluxo de informações minimas exigidas, através dos instrumentos padronizados pelo sistema. As unidades de saúde do Estado, então municipalizadas, foram "engolidas" pela assistência médica, modificando o seu caráter e perfil de atuação hegemônico nas ações de prevenção que as caracterizavam anteriormente.

Este choque entre as chamadas medicina curativa $X$ medicina preventiva, no âmbito dos serviços de saúde dos Municipios, determinou inúmeras resistências ao processo. Dentre elas, vale ressaltar as dificuldades na investigação de casos suspeitos de meningite, ou difteria onde é necessário o exame clínico dos comunicantes através da visita à residência do suspeito, o que exige deslocamento de médicos para fora da unidade; a capacitação e adequação dos serviços, em especial a formação de profissionais para o atendimento de tuberculose e hanseniase; adequação do laboratório e a organização do fluxo de envio de amostras para o Instituto Adolfo Lutz; o conhecimento epidemiológico dos problemas de saúde existentes e a priorização dos programas de intervenção, segundo a lógica do planejamento e não de acordo com a demanda apenas; a hierarquização dos serviços e a organização da referência e contra-referência, entre outros.

Este choque resultou numa predominância da assistência médica individual curativa sobre a programação de saúde, dando ao processo o ritmo da pressão da demanda. O reforço nesta linha de direção veio no financiamento das ações, já que se passou a cobrar e receber por procedimento.

Para melhor dimensionar a situação dos municipios em relação ao financiamento, Pimenta, A. L. (5) diz referindo-se a Norma Operacional Básica $n^{\circ} 1$ : 
"Com esta norma os municipios passam a ser tratados como mero prestadores de serviços, e as ações são remuneradas de acordo com as prioridades definidas pelo próprio INAMPS. Passamos -os Secretários Municipais de Saúde- a viver a chamada "ditadura da tabela" com uma total distorção de toda a proposta de o municipio assumir a gestão do Sistema de Saúde no nivel local: as ações melhor remuneradas pela tabela são exatamente aquelas que envolvem alta tecnologia e são de caráter curativo e individual, sendo as ações coletivas mal remuneradas, ou até mesmo não remuneradas".

$\mathrm{Na}$ esfera estadual, a área responsável pela Saúde Coletiva demonstra sua preocupação. Como define o texto "Reflexos sobre as ações de saúde coletiva" apresentado no Seminário de Dirigentes da Secretaria Estadual de Saúde:

"É importante salientar que os mecanismos atualmente vigentes (Lei $8142 / 90$ e Norma Básica operacional 01/91 do M.S. , reeditada pela Resolução 273 de $17 / 7 / 91$ para repasse de recursos da União para os Estados e Municipios) têm como único princípio o critério populacional e como parâmetro de alocação a produção de serviços realizados para todas as ações de saúde. Isto significa que todos os serviços (de assistência médica ou de saúde coletiva) receberão recursos mediante a "prestação de serviços".(9)

O quadro delineado até aqui coloca o Sistema de Vigilâncıa Epidemiológica e o CVE que o coordena numa posição incômoda, pois seus "braços", os niveis regionais, estavam despreparados e suas "pernas", as unidades de saúde, transformadas e gerenciadas para uma direção diferente da sua. Esta caricatura demonstra o descompasso e evidencia o distanciamento entre os diversos niveis do sistema. 
A incorporação da Vigilância Epidemiológica tem sido gradativa nos Municípios. No final de 1993, o Centro de Vigilância Epidemiológica atualizou as informações sobre a municipalização das ações sob sua coordenação, através de um questionário enviado aos ERSAs, excluídos aqueles pertencentes à capital(10). Para esta avaliação padronizou-se uma classificação dos municípios segundo estágios e desenvolvimento das ações de Vigilância Epidemiológica(11). Neste estudo (10), chama a atenção que $1,5 \%$ dos municípios do Estado não executaram nenhuma ação de vigilância (estágio $O-A, O-B$ ) e que aproximadamente $4 \%$ apenas (estágio 4B), executam as ações de vigilância de forma autônoma, incluindo análise epidemiológica e/ou treinamento específico. A grande maioria executa as atividades básicas previstas, porém a investigação de surtos e epidemias, a análise epidemiológica e o treinamento ainda estão sob responsabilidade dos ERSAs. As unidades vacinam, notificam, investigam e passam as informações aos ERSAs, inicialmente chamados de SUDS regionais e posteriormente SUS regionais, porém a consistência, consolidação e análise das informações permanecem no Estado, nas equipes de Vigilância regionais e no nivel central do sistema. Ou melhor, a análise epidemiológica que instrumentaliza o diagnóstico e o planejamento em saúde determinando as prioridades de atuação fica na competência do Estado e não no Município. Portanto a descentralização não ocorre por completo e a responsabilidade pela solução dos problemas de saúde daquela população fica restrita à prestação de assistência médica, reduzida à consulta médica e exames subsidiários.

O Município reage cobrando recursos para a execução das ações de saúde coletiva, os quais não estão previstos de forma especifica no repasse de verbas. A discussão entre Estado e Município na área de Saúde Coletiva, em especial na Vigilância Epidemiológica, traduz-se numa cobrança mútua, em que a 
temática são viaturas, recursos humanos capacitados, fluxo de papéis, fluxo de exames e máquinas para controle de vetores. Uma conversa sem fim, onde um Estado pobre conversa com um Municipio atolado de problemas, com uma Federação vilã. A população continua sem perspectiva de receber do serviço público assistência à saúde de qualidade; sem compreender em nenhum momento, a quem pertence o mosquito, o esgoto ao lado da sua casa, ou porque este serviço atende determinada doença e aquele outro não. Para a população há sempre um culpado que ora é o prefeito, ora o governador, ora o presidente, dificultando ainda mais a "famosa" participação e conscientização popular.

\section{PROPOSTAS}

Para que seja superado o impasse existente é importante retomar a conversa, refazer o diálogo e direcionar a discussão na linha da descentralização, hierarquização, redefinindo os papéis de Estado e Município, recuperando a análise epidemiológica como base para o planejamento de saúde. Neste sentido propomos que:

1. Os níveis regionais devem estar capacitados para serem legítimos interlocutores técnicos com os Municipios. É necessário resgatar seu papel no acompanhamento epidemiológico dos Municípios e na construção de propostas e políticas de intervenção no nivel regional, de acordo com áreas homogêneas de risco de transmissão das doenças sob vigilância.

2. O sistema de informação de doenças de notificação compulsória deve estar voltado para a capacitação dos Municípios nas normas e procedimentos da vigilância e na consistência, consolidação e 
análise das informações produzidas, garantindo a estes o ônus e a responsabilidade da eleição das estratégias de intervenção.

3. A construção de propostas e políticas de intervenção deve ser feita em conjunto (Estado e Municipio) de forma a caracterizar o efetivo repasse de atribuições no bojo da discussão técnica.

4. O repasse de recursos, se necessário, deverá ser o resultado do processo de elaboração da proposta técnica conjunta e definido dentro das prioridades do Municipio.

5. As experiências positivas vividas por alguns Municipios do Estado devem contribuir para a readequação do Estado, e para tanto tem que ser estudadas trazendo para dentro do Estado um novo olhar revigorando e mudando a "cabeça" do sistema de vigilância.

Para atingir estes objetivos e consubstanciar estas propostas em prática existem alguns entraves, quais sejam:

- Os Municípios em geral têm tido, nos últimos anos, uma política salarial adequada com niveis próximos ao mercado. Em contrapartida o Estado conseguiu reduzir os salários dos servidores a um patamar inadministrável, de tal sorte que houve e continua ocorrendo intenso fluxo de quadros do Estado para os Municipios. Isto tem como saldo uma melhoria dos quadros técnicos lotados no Município, o que em si facilita mas não garante a efetivação das propostas. Porém, no nivel do Estado, tem determinado um esvaziamento das equipes regionais e centrais, 
particularmente de técnicos altamente especializados e experimentados que não são rápida e facilmente repostos.

- A Secretaria de Saúde precisa investir na descentralização do sistema, isto é, há que discutir a adequação do perfil dos recursos humanos e o papel dos niveis regionais, capacitando-os para realizarem a supervisão e o acompanhamento dos Municipios, propiciando a estes autonomia na consistência e consolidação das informações, análise epidemiológica e elaboração de propostas voltadas à sua realidade. Com isso, pode ser necessário, também no âmbito do C.V.E., reduzir a quantidade de informações e talvez, num primeiro momento, perder o detalhamento das análises, de forma a estar aberto à construção de um modelo novo, não completamente diferente do anterior, mas mais flexivel e dinâmico. Além disso faz-se necessário estar aberto e vigilante para a compreensão e análise da temática denominada de "Vigilância à Saúde"(12, 13, 14, 15, 16 e 17); este debate foi revigorado pela experiência do Municipio de São Paulo, no periodo de 1989 a 1992 e traz no seu âmago a epidemiologia como base para a programação em saúde e determinante do planejamento no nivel local. Tal proposta refaz, noutro patamar, as considerações e os princípios que nortearam a criação do Sistema de Vigilância Epidemiológica e o CVE.

No sentido de colaborar para o desenrolar deste processo sugerimos alguns pontos de discussão que nos parecem importantes para a interlocução entre Estado e Municipio na área de Saúde Coletiva, em especial na Vigilância 
Epidemiológica. Apresentamos como exemplo o roteiro utilizado pelo grupo técnico interinstitucional designado para elaborar o Programa de Prevenção e Controle de Dengue e Febre Amarela - Municipio de São Paulo(18).

1. Diagnóstico da situação epidemiológica do Município, considerando o quadro atual do programa e das ações que vem sendo desenvolvidas.

2. Elaboração de proposta técnica conjunta:

Etapa do diagnóstico, procurando identificar áreas de risco homogêneas dentro do Município;

Elaboração de estratégias diferenciadas e programas de controle;

Organização do sistema de vigilância epidemiológica:

- Porta de entrada dos casos no Município - eleição de serviços sentinela

- Diagnóstico laboratorial

- Notificação e investigação epidemiológica

- Fluxo de informação dos casos, consolidação e análise dos dados no nivel regional e central do Municipio.

- Fluxo de informação dos casos para os niveis regionais e central da SES. 
3. Diagnóstico da estrutura existente para a operacionalização das atividades:

- Recursos humanos

- Recursos de materiais

4. Relacionamento das necessidades de recursos humanos, materiais e de capacitação para operacionalização das atividades e a estratégia a ser utilizada para descentralização do programa.

5. Discussão com o Município da planilha a ser utilizada pelo Estado para acompanhamento supervisão e avaliação das atividades desenvolvidas no Municipio.

\section{AGRADECIMENTOS}

Agradecemos a Marina Ruiz de Matos pela leitura critica do manuscrito e pelas valiosas e calorosas sugestões. 


\section{REFERÊNCIAS BIBLIOGRÁFICAS}

1. PERRENOUD, B. A. F. et al O sistema de vigilância epidemiológica sob a crise do setor saúde. Saúde e Soc, 1(1): 15-23, 1992.

2. CARVALHO, G. C. M. O momento atual do SUS: a ousadia de cumprir e fazer cumprir a lei. Saúde e Soc, 2(1): 9-24,1993.

3. CARVAlHO, G. I. Sistema Único de Saúde: comentário à Lei Orgânica de Saúde, São Paulo, Ed. HUCITEC, 1992.

4. GUEDES, J. S. et al Considerações acerca do processo de criação dos sistemas unificados e decentralizados de saúde (SUDS) agosto/1987. [mimeo].

5. PIMENTA, A. L. O SUS e a municipalização: a luz da experiência concreta. Saúde e Soc., 2(1): 25-40, 1993.

6. SECRETARIA DE ESTADO DA SAÚDE DE SÃO PAULO. Centro de Informações de Saúde. Manual de vigilância epidemiológica: normas e instruções. São Paulo, 1978.

7. WALDMAN, E. A. Vigilância epidemiológica como prática de saúde pública. São Paulo, 1991 [Tese de Doutorado - Faculdade de Saúde Pública da Universidade de São Paulo].

8. Diário Oficial, Decreto 24565 de 27/12/85.

9. SEMINÁRIO DE DIRIGENTES DA SECRETARIA ESTADUAL DE SAUUDE/CONFERÊNCIA ESTADUAL DE SAÚDE, 1\%/CONFERÊNCIA NACIONAL DE SAÚDE, 9a , São Paulo, 1991. Reflexões sobre as ações de saúde coletiva. São Paulo, CVE/CVS/SUCEN/IAL, 1991

10. Centro de Vigilância Epidemiológica (CVE). Municipalização das ações de vigilância e epidemiológica. São Paulo, 1994. [mimeografado]

11. Centro de Vigilância Epidemiológica (CVE). Sistema de vigilância epidemiológica (SVE) no Estado de São Paulo. São Paulo, 1994 [mimeografado] 
12. UNGLERT, C. V. S. et al. Acesso aos serviços de saúde: uma abordagem de geografia em saúde pública. Rev. Saúde Pública., 21: 439-46, 1987.

13. VILASBÔAS, A. L. et al. A Vigilância à saúde no distrito sanitário. Brasilia, OPAS/OMS, 1992. (Série Desenvolvimento de Serviços de Saúde n 10)

14. GOLDBAUM, M. Vigilância da saúde. In: Seminário Nacional de Vigilância Epidemiológica, Brasilia, 1993. Anais. Brasilia, FNS/CENEPI.

15. BARATA, R. B. Reorientação das práticas de vigilância epidemiológica. in: Seminário Nacional de Vigilância Epidemiológica, Brasilia, , 1993. Anais. Brasilia. FNS/CENEPI

16. WALDMAN, E. A. As concepções de vigilância como instrumento de saúde pública e a implantação do SUS. In: Seminário Nacional de Vigilância Epidemiológica, Brasilia, 1993. Anais. Brasilia, FNS/CENEPI

17. SILVA, L. J. Vigilância epidemiológica: uma proposta de transformação. Saúde e Soc, 1(1): 7-14, 1992.

18. SCHOUT, D.; KATZ, G.; PARODI, E. S. M.; GLASSER, C. M.; LO. S. S.; MARCONDES, A. E. C. Programa de prevenção e controle de dengue e febre amarela - Município São Paulo. São Paulo, 1993. [mimeografado]. 